\title{
Development and validation of an UPLC-MS/MS method for the determination of 7-hydroxymitragynine, a $\mu$-opioid agonist, in rat plasma and its application to a pharmacokinetic study
}

\author{
Pradeep K. Vuppala ${ }^{a}$, Seshulatha Jamalapuram ${ }^{\mathrm{a}}$, Edward B. Furr ${ }^{\mathrm{b}}$, Christopher R. \\ McCurdy, ${ }^{b, c}$, and Bonnie A. Avery ${ }^{*}, a$ \\ aDepartment of Pharmaceutics, The University of Mississippi, University, MS 38677 USA \\ bDepartment of Medicinal Chemistry, The University of Mississippi, University, MS 38677 USA \\ 'Department of Pharmacology, School of Pharmacy, The University of Mississippi, University, MS \\ 38677 USA
}

\begin{abstract}
A simple, sensitive and specific ultra-performance liquid chromatography-tandem mass spectrometry (UPLC-MS/MS) method has been developed and validated to determine the concentrations of 7-hydroxymitragynine in rat plasma. Following a single step liquid-liquid extraction of plasma samples using chloroform, 7-hydroxymitragynine and the internal standard (tryptoline) were separated on an Acquity UPLC ${ }^{\mathrm{TM}}$ BEH C $\mathrm{C}_{18}(1.7 \mu \mathrm{m}, 2.1 \mathrm{~mm} \times 50 \mathrm{~mm})$ column using an isocratic elution at a flow rate of $0.2 \mathrm{~mL} / \mathrm{min}$. The mobile phase consisted of $0.1 \%$ acetic acid in water and $0.1 \%$ acetic acid in acetonitrile $(10: 90, v / v)$. The run was $2.5 \mathrm{~min}$. The analysis was carried out under the multiple reaction-monitoring mode using positive electrospray ionization. Protonated ions $[\mathrm{M}+\mathrm{H}]^{+}$and their respective product ions were monitored at the following transitions: 415>190 for 7-hydroxymitragynine and 173>144 for the internal standard. The calibration curve was linear over the range of 10 to $4000 \mathrm{ng} / \mathrm{mL}\left(r^{2}=0.999\right)$ with a lower limit of quantification (LLOQ) of $10 \mathrm{ng} / \mathrm{mL}$. The extraction recoveries ranged from $62.0 \%$ to $67.3 \%$ at concentrations $(20,600,3200 \mathrm{ng} / \mathrm{mL})$. Intra- and inter-day assay precisions (relative standard deviation) were less than $15 \%$ and the accuracy was within $96.5 \%$ to $104.0 \%$. This validated method was successfully applied to quantify 7-hydroxymitragynine in rat plasma following intravenous administration.
\end{abstract}

\section{Introduction}

Morphine is a well-known and widely used analgesic for the clinical treatment of acute and chronic severe pain. Although very popular, it has a number of adverse effects: respiratory depression, nausea, vomiting, constipation, tolerance and dependence. In order to develop powerful analgesics without side effects, many morphine-related derivatives were synthesized but most of the derivatives that are in use clinically still suffer from the side effects similar to those possessed by morphine. The primary research objectives in the

"Corresponding author: Dr. Bonnie A. Avery, Department of Pharmaceutics, School of Pharmacy, The University of Mississippi, University, MS 38677, Phone: +1 662-915-5163, Fax: +1 662-915-1177, bavery@olemiss.edu. 
opioid area are to understand the underlying biology of the endogenous opioid systems, discovery of new analgesic compounds devoid of unwanted effects associated with morphine, and to develop new therapies for the treatment of opioid addiction (Corbett et al., 2006).

The leaves of the Mitragyna speciosa tree have long been used in Thailand and Malaysia for their opium- and coca-like effects, and as a replacement for opium. In addition, the aqueous extracts of leaves have been used as a traditional medicine for common illnesses such as coughing, fever, diarrhea, muscle pain, hypertension, and also to treat opium addiction (Matsumoto et al., 2004; Babu et al., 2008; Vuppala et al., 2011). This medicinal plant contains many indole-alkaloids. Mitragynine, a corynanthine-like alkaloid, is the main constituent present in leaves (66\% of the alkaloid content) (Takayama et al., 2001; Adkins et al., 2011). Though, the structure of mitragynine is different from morphine, in pharmacological experiments mitragynine showed agonistic effects on opioid receptors (Watanabe et al., 1997). Watanabe et al., compared the antinociceptive effect of Mitragyna speciosa and mitragynine in in vivo experiments, but the antinociceptive effect of mitragynine was less potent than that of the crude extract of Mitragyna speciosa. This finding suggests that minor constituents of the Mitragyna speciosa extract might have a very potent antinociceptive effect (Watanabe et al., 1999). Horie et al. studied the opioid agonistic effects of the constituents of Mitragyna speciosa using in vitro assays (Horie et al., 2005). Among them, 7-hydroxymitragynine (7-OHMG), an oxidized form of mitragynine (a-hydroxyl group at the $\mathrm{C} 7$ position) showed the most potent effect. This suggests that the more potent opioid effect of the Mitragyna speciosa extract may be due to the activity of 7OHMG (Matsumoto et al., 2004; Horie et al., 2005). Matsumoto et al., demonstrated that, subcutaneous or oral administration of 7-OHMG $(2.5-10 \mathrm{mg} / \mathrm{kg})$ to mice induced a potent and dose dependent antinociceptive effect in the tail-flick and hot-plate tests. These effects were more potent than that of morphine when subcutaneously or orally administered (Matsumoto et al., 2004; Babu et al., 2008). In guinea-pig ileum, 7-OHMG inhibited electrically induced contraction through the opioid receptors. Isolated tissue studies and receptor binding assays revealed that 7-OHMG has a higher affinity for $\mu$-opioid receptors relative to the $\delta$ - and $\kappa$ - receptors (Matsumoto et al., 2004; Matsumoto et al., 2006).

Furthermore, 7-OHMG was found to be 4.9-6.4 times less constipating than morphine at equi-antinociceptive doses (Matsumoto et al., 2006). Matsumoto et al., attempted to overlay the 7-OHMG with morphine using molecular modeling techniques but were not successful. Therefore, it was concluded that 7-OHMG might be interacting with opioid receptors in a different fashion than morphine. These authors also investigated the rewarding effects of 7OHMG using the place-conditioning test in mice. At $1 \mathrm{mg} / \mathrm{kg}$ dose, 7-OHMG showed a slight place preference and at $2 \mathrm{mg} / \mathrm{kg}$ dose, it showed a strong place preference, this indicates that 7-OHMG could have potential for abuse and addiction. The above studies suggest that 7-OHMG is not only the opioid analgesic component but also potentially the reason that Mitragyna speciosa is utilized for psychoactive effects (Matsumoto et al., 2005). Interestingly, the amount of 7-OHMG that is present in Mitragyna speciosa can be greater than $1 \mathrm{mg} / \mathrm{kg}$ when decoctions are made for human consumption. Having a novel structural scaffold for opioid receptor affinity and activity, 7-OHMG lends itself to further investigation as a novel compound for opioid studies. Analogues of 7-OHMG (ethylene 
glycol-bridged and C10-fluorinated derivative, MGM-9) have been synthesized and research is going on to develop a novel analgesic without negative side effects (Matsumoto et al., 2008). Since 7-OHMG possesses interesting pharmacological activities, detection and determination methods for this compound in biological systems are required to facilitate further studies of its pharmacokinetics. One quantitative and two qualitative analytical methods have been reported for 7-OHMG. Hanajiri et al., developed a method for the quantification of 7-OHMG in raw materials and commercial products of Mitragyna speciosa using LC/MS (Kikura-Hanajiri et al., 2009). Leon et al., isolated the 7-OHMG from the leaves of Mitragyna speciosa using thin layer chromatography (Leon et al., 2009). Recently, Le et al., developed a qualitative analytical method using LC/MS/MS for the identification of 7-OHMG in human urine samples (Le et al., 2012). None of the previously published methods are useful for the extraction and quantification of 7-OHMG from plasma samples. In order to facilitate the quantitation of 7-OHMG in pharmacokinetic studies, we developed a simple, sensitive UPLC-MS/MS method in rat plasma. The developed method was validated according to the US-FDA bioanalytical method validation guidance. To our knowledge this is the first bioanalytical method developed for the determination of 7 OHMG in rat plasma.

\section{Experimental}

\section{Materials}

7-OHMG was synthesized from mitragynine by Dr. Christopher R. McCurdy's research group, Department of Medicinal Chemistry, University of Mississippi, as previously published (Takayama et al., 2002; Ponglux et al., 1994). 7-OHMG was analyzed by ${ }^{1} \mathrm{H}$ NMR, ${ }^{13} \mathrm{C}$ NMR, elemental analysis, HPLC and HR-MS, and was found to be $299 \%$ pure. Tryptoline (1,2,3,4-Tetrahydro-9H-pyrido[3,4-b]indole), was used as the internal standard (IS), and was purchased from Sigma-Aldrich (St. Louis, MO, USA). Acetonitrile, chloroform, water and acetic acid were supplied by Fisher Scientific (Fair Lawn, NJ, USA). The solvents used during this study were all HPLC grade. Rat plasma with sodium heparin was purchased from Innovative Research (Peary Court Novi, MI, USA).

\section{Liquid chromatography and mass spectrometry}

A Waters Acquity ultra-performance liquid chromatography (UPLC) system (Milford, MA, USA) equipped with a binary solvent manager, vacuum degasser, thermostatted column compartment, and an auto sampler was used for the quantitation of the compounds. All chromatographic separations were performed using an Acquity UPLC ${ }^{\text {TM }}$ BEH $\mathrm{C}_{18}$ column $(1.7 \mu \mathrm{m}, 2.1 \mathrm{~mm} \times 50 \mathrm{~mm})$ maintained at $25^{\circ} \mathrm{C}$. The isocratic mobile phase, a mixture of $0.1 \%$ acetic acid in water and $0.1 \%$ acetic acid in acetonitrile $(10: 90, v / v)$, was delivered at a flow rate of $0.2 \mathrm{~mL} / \mathrm{min}$ into the electrospray ionization chamber of the mass spectrometer.

Quantification of 7-OHMG and the internal standard was achieved with MS/MS detection using a Waters Micromass Quattro Micro ${ }^{\mathrm{TM}}$ triple quadrupole system (Manchester, UK) equipped with an electrospray interface unit. The system was controlled by MassLynx software version 4.1. The mass spectrometer was operated in the positive electrospray ionization (ESI) mode. The capillary voltage of the ESI was $4.06 \mathrm{kV}$. The temperature of the 
source and desolvation system was maintained at 120 and $200{ }^{\circ} \mathrm{C}$. Nitrogen was used as the nebulizer gas and argon was used as the collision gas. The cone voltage and collision energy were maintained at $23 \mathrm{~V}$ and $27 \mathrm{eV}$, respectively. The detection of the ions was carried out in the multiple reaction-monitoring mode (MRM). The precursor-to-product ion transitions were monitored at $m / z, 415>190$ for $7-O H M G$ and at $m / z, 173>144$ for the IS.

\section{Calibration standards and quality control samples}

The primary stock solutions of 7-OHMG and the IS were prepared in acetonitrile at a concentration of $1 \mathrm{mg} / \mathrm{mL}$. For the working solutions, a stock solution of 7-OHMG was suitably diluted with water : acetonitrile $(50: 50, v / v)$ to obtain concentrations in a range of 100 to $40,000 \mathrm{ng} / \mathrm{mL}$. A working solution of the IS was prepared at a concentration of 1 $\mu \mathrm{g} / \mathrm{mL}$ in water : acetonitrile $(50: 50, v / v)$. The calibration standards were prepared by spiking blank rat plasma with a working standard solution to yield 7-OHMG concentrations of 10, 50, 100, 500, 1000, 2000, 3000 and $4000 \mathrm{ng} / \mathrm{mL}$. Quality control (QC) samples were prepared in the same way as the calibration standards, at concentrations of 20,600, and 3200 $\mathrm{ng} / \mathrm{mL}$, representing low, medium and high concentrations of the calibration curve, respectively.

\section{Sample preparation}

Rat plasma samples (obtained from pharmacokinetic study) stored at $-20{ }^{\circ} \mathrm{C}$ were thawed at ambient temperature before processing. The rat plasma $(100 \mu \mathrm{L})$ samples were spiked with $10 \mu \mathrm{L}$ of the IS working solution $(1 \mu \mathrm{g} / \mathrm{mL})$ and vortexed for $30 \mathrm{~s}$ in a microcentrifuge tube. Liquid-liquid extraction was chosen for the sample preparation. The extraction solvent, chloroform $(800 \mu \mathrm{L})$, was added to the mixture and vortexed for $10 \mathrm{~min}$ on a multi sample holder VWR signature ${ }^{\mathrm{TM}}$ pulsing vortex mixer (VWR, CO, USA). After vortexing, the mixture was centrifuged at $10,000 \times g$ for $10 \mathrm{~min}$. A $750 \mu \mathrm{L}$ aliquot of the organic phase was then carefully transferred with a micropipette into an eppendorf tube and evaporated to dryness in a vacuum oven (Precision Scientific, VA, USA) at $25^{\circ} \mathrm{C}$. The residue was reconstituted in $100 \mu \mathrm{L}$ of mobile phase. A $10 \mu \mathrm{L}$ sample was injected into the UPLCMS/MS system for quantitation. All of the QC samples were prepared following the above method.

\section{Method validation}

A thorough and complete method validation of 7-OHMG in rat plasma was performed as per the United States Food and Drug Administration (US-FDA) Bioanalytical Method Validation Guidance (Jamalapuram et al., 2013; US Food and Drug Administration, 2001; Mandal et al., 2012).

\section{Selectivity}

The selectivity of the developed method was investigated for the assessment of potential interferences at the retention times of 7-OHMG and the IS from endogenous substances. Selectivity was investigated by comparing the chromatograms of six different lots of blank rat plasma, with the corresponding spiked plasma samples with 7-OHMG and the IS. 


\section{Recovery and matrix effect}

The extraction recoveries of 7-OHMG and the IS were determined at low, medium and high QC concentrations $(20,600$, and $3200 \mathrm{ng} / \mathrm{mL})$. The extracted QC $(n=6)$ samples were analyzed and the peak area ratios were compared with peak area ratios of the standards prepared using the mobile phase. The recovery of the analyte and IS need not be $100 \%$, but should be consistent, and reproducible at different concentration levels.

The matrix effects regarding the ionization of 7-OHMG and the IS were determined at the three QC levels (20, 600 and $3200 \mathrm{ng} / \mathrm{mL}$ ). To determine the matrix effect, six different lots of blank rat plasma samples were used to prepare the QC samples $(n=6)$. The matrix effect was evaluated by comparing the average peak areas of the analyte in post extraction spiked samples to those obtained from corresponding standards injected directly into the mobile phase.

\section{Linearity and lower limit of quantification}

Linearity was determined in the range of $10-4000 \mathrm{ng} / \mathrm{mL}$ by plotting the peak area ratio of 7OHMG to IS versus the nominal concentrations of 7-OHMG in plasma. The sensitivity of the assay was determined using the lower limit of quantification (LLOQ). This is the lowest concentration on calibration curve that produced a signal to noise ratio $(S / N)$ of 10 , with a precision of $\_20 \%$ relative standard deviation (\%RSD) and accuracy within $\pm 20 \%$. The LLOQ was evaluated by analyzing six replicates of plasma samples at LLOQ level on three separate days. System suitability tests were performed to verify the adequate working of the equipment. The test was carried out using six replicates of extracted LLOQ samples injected immediately prior to each validation and study sample analysis runs.

\section{Accuracy and precision}

Intra- and inter-day accuracy and precision were evaluated by analyzing six replicates of the QC samples at three different concentrations (20, 600 and $3200 \mathrm{ng} / \mathrm{mL}$ ). For intra-day assay accuracy and precision, QC samples were extracted and analyzed using a freshly prepared calibration curve on the given day; for inter-day assay accuracy and precision, the same procedure repeated over three consecutive days. The accuracy was expressed as bias $\%$, i.e. (measured concentration-actual concentration)/(actual concentration) $\times 100 \%$, and the precision as relative standard deviation (\%RSD). The evaluation of precision was based on the criteria that the RSD for each concentration should be $₫ 5 \%$. Similarly, accuracy should not deviate by $\pm 15 \%$ of the actual concentration.

\section{Stability}

The stability of 7-OHMG in rat plasma was evaluated using QC samples (20, 600 and 3200 $\mathrm{ng} / \mathrm{mL}$ ) in six replicates. The stability of 7-OHMG was tested under the following conditions: (1) freeze-thaw stability in rat plasma through three freeze-thaw cycles (2) shortterm stability in rat plasma at room temperature for $12 \mathrm{~h} \mathrm{(3)} \mathrm{long-term} \mathrm{stability} \mathrm{in} \mathrm{rat} \mathrm{plasma}$ stored at $-20^{\circ} \mathrm{C}$ for 30 days (4) post-preparative stability during storage in the auto sampler at $25{ }^{\circ} \mathrm{C}$ for $12 \mathrm{~h}$. To determine freeze-thaw stability, QC samples were stored at $-20{ }^{\circ} \mathrm{C}$ for $24 \mathrm{~h}$, thawed at room temperature and refrozen for 12-24 h under the same conditions. This 
procedure was repeated for three cycles. At the end of cycles the stability samples were processed, analyzed and the results were compared with the freshly prepared QC samples. For the short-term temperature stability, QC samples $(n=6)$ were stored at room temperature for $12 \mathrm{~h}$. The samples were analyzed after $12 \mathrm{~h}$ and the results were compared with the freshly prepared QC samples. For long-term stability, QC samples $(n=6)$ were stored at -20 ${ }^{\circ} \mathrm{C}$ for a period of 30 days. The samples were processed and analyzed on the $30^{\text {th }}$ day and the results were compared with the freshly prepared QC samples. The post-operative stability (auto-sampler stability) was assessed by re-analyzing the samples that were kept in the autosampler at $25^{\circ} \mathrm{C}$ for $12 \mathrm{~h}$.

\section{Application to a pharmacokinetic study on rats}

Animal experiments were performed in accordance with the guidelines of the Institutional Animal Care and Use Committee (IACUC) of the University of Mississippi. Male SpragueDawley rats ( $n=6$ ) weighing 240-250 $g$ obtained from Harlan Laboratories (Indianapolis, IN, USA) were used to determine the in vivo pharmacokinetics of 7-OHMG following intravenous (IV) administration. The rats were initially housed the institutional vivarium for $48 \mathrm{~h}$. The vivarium is controlled environment with the temperature maintained between $21-23{ }^{\circ} \mathrm{C}$, humidity $50 \pm 10 \%$, and programmed for a $12 \mathrm{~h}$ light/dark cycle. Prior to dosing the animals, they were moved to the procedure room. The rats were fasted for $12 \mathrm{~h}$ before dosing and for the first $4 \mathrm{~h}$ after dosing but had free access to water during the entire experiment. The intravenous (IV) formulation was prepared using $10 \%$ polyethylene glycol 400 in water and was dosed to rats at $4 \mathrm{mg} / \mathrm{kg}$. The IV formulation was prepared on the day of dosing and was administered $(0.2 \mathrm{~mL})$ as IV bolus in $30 \mathrm{~s}$ through the jugular vein cannula. After that, the cannula was flushed with heparinized saline $(0.2 \mathrm{~mL})$ to ensure complete administration of the dose. Blood samples $(0.18 \mathrm{~mL})$ were collected through each rat's indwelling cannula at intervals of $0,2,5,10,15,30,45,60,90,120,180,240$ and 480 min post IV dose. After each blood sampling, catheter was flushed with $0.18 \mathrm{~mL}$ of heparinized saline solution ( 250 units $/ \mathrm{mL}$ ). The blood samples were put into a heparinized microcentrifuge tube and immediately centrifuged at $10,000 \times g$ for 10 min at $4{ }^{\circ} \mathrm{C}$. An aliquot $(100 \mu \mathrm{L})$ of plasma was transferred into $1.5 \mathrm{~mL}$ microcentrifuge tube and then stored at $-20{ }^{\circ} \mathrm{C}$ until analysis.

\section{Results and discussion}

\section{Liquid chromatography and mass spectrometry}

The ionization and fragmentation of 7-OHMG and the IS were obtained by infusing their respective solutions $(10 \mu \mathrm{g} / \mathrm{mL})$ into the electrospray injection unit of a mass spectrometer at a constant flow rate of $10 \mu \mathrm{L} / \mathrm{min}$ using an integrated syringe pump. Mass parameters were tuned in both positive and negative ionization modes. However, the response observed in positive mode was much higher for both compounds compared to that of negative mode. Both the analyte and IS showed predominant protonated molecular ion at $\mathrm{m} / \mathrm{z}$ of 415 and 173 for 7-OHMG and IS, respectively in Q1 spectrum. The protonated form of the analyte and IS, $[\mathrm{M}+\mathrm{H}]+$ ion, were used as the precursor ion to obtain the product ion spectra. The fragmentation was initiated using argon gas for collision activated dissociation to break the precursor ions. The most abundant ions found in the Q3 product ion spectra were at m/z 190 
and 144 for 7-OHMG and IS, respectively. The MS/MS transition of 415>190 for 7-OHMG and 173>144 for the IS were selected for identification and quantitation. Chemical structures, protonated molecular ions spectra of 7-OHMG and the IS and their respective product ions spectra are shown in Figure 1, Figure 2 and Figure 3.

Because 7-hydroxymitragynine is a non-polar compound we evaluated commercially available RPLC columns such as $\mathrm{C}_{18}$ and $\mathrm{C}_{8}$ and Atlantis. A double peak was observed with $\mathrm{C}_{8}$ column and peak shape on Atlantis column was tailed. After a series of trials, $\mathrm{C}_{18}$ column was considered to be optimal. 7-OHMG retained well with good peak shapes on Waters Acquity UPLC ${ }^{\text {TM }}$ BEH C 18 column $(1.7 \mu \mathrm{m}, 2.1 \mathrm{~mm} \times 50 \mathrm{~mm})$. The main advantage of using UPLC over HPLC was a significant decrease of analysis time and reduction in solvent consumption (Novakova et al., 2006; Vuppala et al., 2011). The UPLC system with $1.7 \mu \mathrm{m}$ particle size column reduces analysis time up to nine times comparing to the conventional system using $5 \mu \mathrm{m}$ particle packed analytical columns (Novakova et al., 2006). In our method the use of UPLC with $1.7 \mu \mathrm{m}$ particle size column resulted in a retention time of 0.5 and $0.4 \mathrm{~min}$ for 7-OHMG and the IS, respectively. To obtain efficient ionization responses for 7-OHMG and the IS, various mobile phases were evaluated. 7-OHMG is a basic compound therefore, acidic modifiers (acetic acid and formic acid) were added to obtain a higher ionization and MS/MS response. Different concentrations of acetic acid and formic acid $(0.05 \%, 0.1 \%$ and $0.2 \%)$ in aqueous and organic phases were tested to improve the chromatographic peak shapes and the MS/MS response. The MS/MS response of 7OHMG using acetic acid was about 2 times higher than that obtained with formic acid. Our results indicated that the addition of $0.1 \%$ acetic acid to aqueous phase and organic phase greatly improved the MS/MS response of analyte and the IS. Acetonitrile was chosen as the organic phase as it resulted in a higher analyte response with a lower background noise compared to methanol. The optimized mobile phase consisted of $0.1 \%$ acetic acid in water and $0.1 \%$ acetic acid in acetonitrile $(10: 90, v / v)$ at a flow rate of $0.2 \mathrm{~mL} / \mathrm{min}$. Tryptolin was selected as the internal standard because its chromatographic behavior and extraction efficiency were similar to that of 7-OHMG (Chen et al., 2007; Jamalapuram et al., 2012). A one-step liquid-liquid extraction procedure was used for the sample preparation. Liquidliquid extraction is a simple and cost efficient technique helpful in producing a spectroscopically clean sample and avoiding the introduction of nonvolatile materials onto the column and mass spectrometer. Several extraction solvents (acetonitrile, chloroform, dichloromethane, ethyl acetate and methyl tert-butyl ether) were tested to optimize recovery. Except chloroform, all the solvents showed recovery of less than 50\%. Use of chloroform produced a clean chromatogram for a blank plasma sample and yielded good recovery for the 7-OHMG (64.6 $\pm 5.6 \%)$ and the IS $(71.3 \pm 3.9 \%)$ from the plasma.

\section{Method validation}

\section{Selectivity}

Selectivity is the ability of an analytical method to differentiate and quantify the analyte in the presence of other components in the sample. Comparing the chromatograms of six different lots of blank rat plasma was used to assess selectivity. Chromatograms of the blank plasma and plasma spiked with 7-OHMG and IS are given in Figure 4. In the chromatogram 
display settings of the Masslynx software, the Y- scales were adjusted to $100 \%$ intensity. Therefore, the Y-axis will always be $100 \%$ for all the chromatograms (blank or high concentration sample). The number of ions detected at the peak is shown on the right top corner of the chromatogram. The baseline separation of two adjacent peaks is generally essential in conventional LC with UV detection due the non-specific nature of the detector, especially when wavelengths are used below $250 \mathrm{~nm}$. In case of LC-MS, if the peaks have independent MS signals, complete chromatographic separation is not required (Pitt, 2009). The retention times of 7-OHMG and the IS were 0.5 and $0.4 \mathrm{~min}$, respectively. Endogenous peaks at the retention time of the analytes were not observed for any of the blank rat plasma batches indicating no significant endogenous interference during the plasma sample analysis.

\section{Recovery and matrix effect}

The mean recoveries of 7-OHMG from rat plasma at low, medium, and high QC concentrations were $64.5 \pm 4.3 \%, 62.0 \pm 6.1 \%$ and $67.3 \pm 6.3 \%$, respectively (mean $\pm \mathrm{SD}, n=6$ ). Mean recovery for the IS was $71.3 \pm 3.9 \%$ at $100 \mathrm{ng} / \mathrm{mL}$. The results indicated that the recovery of 7-OHMG was consistent and was not concentration-dependent. The recovery of IS was also consistent and reproducible.

A post-extraction addition technique was used to determine the affect of co-eluting endogenous compounds on ionization efficiency of the method. The matrix effects on recovery of spike-after-extraction samples at $20,600,3200 \mathrm{ng} / \mathrm{mL}$ of 7-OHMG were found to be $100.2 \pm 6.3 \%, 98.6 \pm 4.9 \%$ and $94.1 \pm 6.5 \%$, respectively. The same evaluation was performed on the IS and the recovery was $93.1 \pm 7.3 \%$ at $100 \mathrm{ng} / \mathrm{mL}$. The results showed that the matrix effects from endogenous plasma components on the ionization of 7-OHMG and the IS were negligible.

\section{Linearity and LLOQ}

The peak area ratios of 7-OHMG/IS versus the nominal concentrations of 7-OHMG showed a good linear relationship over the concentration ranges of 10 to $4000 \mathrm{ng} / \mathrm{mL}$ in plasma. A typical regression equation for the calibration curve resulted in an equation of the line of:

$$
y=0.0123 x+0.0020\left(r^{2}=0.999\right)
$$

Where $y$ represents the peak area ratios of 7-OHMG to the IS and $x$ represents plasma concentrations of analyte. The \%RSD of slope and the intercept were 2.7 and $5.6(n=6)$, respectively. All correlation coefficients of the curves were found to be greater than 0.99 . The LLOQ in rat plasma was $10 \mathrm{ng} / \mathrm{mL}$ with a precision (\%RSD) and accuracy of $5.2 \%$ and $103 \%$, respectively. The limit of detection was found to be $2 \mathrm{ng} / \mathrm{mL}$. In system suitability samples runs peak retention time, peak shape, and absolute response were consistent and the precision (\%RSD) of system suitability samples was found to be $4.4 \%$. 


\section{Accuracy and precision}

Intra- and inter-day accuracy and precision were determined from the analysis of QC samples $(n=6)$ at concentrations of 20,600 , and $3200 \mathrm{ng} / \mathrm{mL}$. The intra- and inter-day precision and accuracy data at three QC concentrations of 7-OHMG were listed in Table 1. The precision was determined as the \%RSD and the accuracy was expressed as bias \% (percentage of the measured concentration over the actual concentration). The intra-day precision (\%RSD) ranged from $1.0 \%$ to $2.2 \%$, and the inter-day precision ranged from $2.1 \%$ to $4.1 \%$. The intra-day accuracy (bias $\%$ ) ranged from $-1.5 \%$ to $4.0 \%$ and the inter-day accuracy ranged from $-3.5 \%$ to $2.0 \%$. According to the US-FDA bioanalytical method validation guidance, the precision determined at each concentration level should not exceed $15 \%$ of RSD except for the LLOQ, where it should not exceed $20 \%$ of the RSD. For accuracy, the bias $\%$ value should be within $\pm 15 \%$ except at LLOQ, where it should be within $\pm 20 \%$. As all these values were found to be within limits specified by US-FDA, the established method has a satisfactory accuracy, precision and reproducibility.

\section{Stability}

The QC samples ( $n=6)$ of 7-OHMG at three concentrations $(20,600,3200 \mathrm{ng} / \mathrm{mL})$ were used for stability experiments. The stability of 7-OHMG was investigated to cover expected conditions during sample preparation, storage and processing of all samples. Stability studies performed were freeze-thaw, short-term temperature, long-term and auto-sampler stability. The results (Table 2) indicated that 7-OHMG was stable in plasma, for three freeze-thaw cycles when stored at $-20{ }^{\circ} \mathrm{C}$ and thawed to room temperature, for $12 \mathrm{~h}$ at room temperature, and for 30 days at $-20^{\circ} \mathrm{C}$. Reconstituted samples were found to be stable for 12 $\mathrm{h}$ in an auto-sampler which was maintained at $25^{\circ} \mathrm{C}$.

\section{Application to pharmacokinetic Study}

In this study, we explored the intravenous pharmacokinetics of 7-OHMG in SpragueDawley rats $(n=6)$ after a single dose of $4 \mathrm{mg} / \mathrm{kg}$. The validated UPLC-MS/MS method was successfully applied in the determination of 7-OHMG concentrations in rat plasma. The plasma concentration-time profiles of a single IV dose of 7-OHMG were shown in Figure 5. A noncompartment model was used to calculate the pharmacokinetic parameters with the WinNonlin 5.2 (Pharsight, CA, USA) software. The results of the IV pharmacokinetic parameters were shown in Tables 3 .

After an IV dose of 7-OHMG, a mean maximum plasma concentration $\left(\mathrm{C}_{\max }\right)$ was found to be $3.0 \pm 0.3 \mu \mathrm{g} / \mathrm{mL}$. The plasma concentration of 7-OHMG decreased rapidly and was eliminated from plasma with a terminal half-life of $22.9 \pm 3.6 \mathrm{~min}$. The initial rapid decline in the plasma concentration indicates that the compound might have left the plasma and been distributed into the other tissues. The volume of distribution and clearance were found to be $1595.8 \pm 586.3 \mathrm{~mL} / \mathrm{kg}$ and $44.2 \pm 14.8 \mathrm{~mL} / \mathrm{min} / \mathrm{kg}$, respectively. The observed high clearance might be the reason for its short half-life but further studies will be conducted to confirm these findings. 


\section{Conclusions}

By optimizing the chromatographic conditions, a sensitive and rapid bioanalytical method was developed for the quantification of 7-OHMG in rat plasma. The present optimized method was validated to guarantee a reliable determination of 7-OHMG in rat plasma. The sample preparation procedure involves a one step liquid-liquid extraction, and the analysis requires only $100 \mu \mathrm{L}$ of plasma. The LLOQ of this method is low enough to meet the needs of pharmacokinetic study of low dose 7-OHMG with good intra-day and inter-day reproducibility. It is the first report of UPLC/MS/MS method on the determination of 7OHMG concentration in vivo so far. The developed method was successfully applied to pharmacokinetic study of 7-OHMG after intravenous administration of $4 \mathrm{mg} / \mathrm{kg}$ dose.

\section{Acknowledgments}

The project described was supported by Grant Number NIGMS P20GM104932 from the National Center for Research Resources. The content is solely the responsibility of the authors and does not necessarily represent the official views of the National Center for Research Resources or the National Institutes of Health.

This investigation was conducted in a facility constructed with support from research facilities improvement program C06 RR-14503-01 from the NIH National Center for Research Resources.

\section{References}

Adkins JE, Boyer EW, McCurdy CR. Mitragyna speciosa, a psychoactive tree from Southeast Asia with opioid activity. Current topics in medicinal chemistry. 2011; 11:1165-1175. [PubMed: 21050173]

Babu KM, McCurdy CR, Boyer EW. Opioid receptors and legal highs: Salvia divinorum and Kratom. Clin Toxicol (Phila). 2008; 46:146-152. [PubMed: 18259963]

Chen L, Qin F, Ma Y, Li F. Quantitative determination of azithromycin in human plasma by ultra performance liquid chromatography-electrospray ionization mass spectrometry and its application in a pharmacokinetic study. Journal of chromatography B, Analytical technologies in the biomedical and life sciences. 2007; 855:255-261.

Corbett AD, Henderson G, McKnight AT, Paterson SJ. 75 years of opioid research: the exciting but vain quest for the Holy Grail. British journal of pharmacology. 2006; 147(Suppl 1):S153-162. [PubMed: 16402099]

Horie S, Koyama F, Takayama H, Ishikawa H, Aimi N, Ponglux D, Matsumoto K, Murayama T. Indole alkaloids of a Thai medicinal herb, Mitragyna speciosa, that has opioid agonistic effect in guinea-pig ileum. Planta medica. 2005; 71:231-236. [PubMed: 15770543]

Jamalapuram S, Vuppala PK, Abdelazeem AH, McCurdy CR, Avery BA. Ultra-performance liquid chromatography tandem mass spectrometry method for the determination of AZ66, a sigma receptor ligand, in rat plasma and its application to in vivo pharmacokinetics. Biomedical chromatography : BMC. 2013

Jamalapuram S, Vuppala PK, Mesangeau C, McCurdy CR, Avery BA. Determination of a highly selective mixed-affinity sigma receptor ligand, in rat plasma by ultra performance liquid chromatography mass spectrometry and its application to a pharmacokinetic study. Journal of chromatography B, Analytical technologies in the biomedical and life sciences. 2012; 891-892:1-6.

Kikura-Hanajiri R, Kawamura M, Maruyama T, Kitajima M, Takayama H, Goda Y. Simultaneous analysis of mitragynine, 7-hydroxymitragynine, and other alkaloids in the psychotropic plant "kratom" (Mitragyna speciosa) by LC-ESI-MS. Forensic Toxicology. 2009; 27:67-74.

Le D, Goggin MM, Janis GC. Analysis of mitragynine and metabolites in human urine for detecting the use of the psychoactive plant kratom. J Anal Toxicol. 2012; 36:616-625. [PubMed: 23024321] 
Leon F, Habib E, Adkins JE, Furr EB, McCurdy CR, Cutler SJ. Phytochemical characterization of the leaves of Mitragyna speciosa grown in U.S.A. Natural product communications. 2009; 4:907-910. [PubMed: 19731590]

Mandal B, Balabathula P, Mittal N, Wood GC, Bhattacharjee H. Development and validation of a spectrofluorimetric method for the determination of erlotinib in spiked human plasma. Journal of fluorescence. 2012; 22:1425-1429. [PubMed: 22875639]

Matsumoto K, Hatori Y, Murayama T, Tashima K, Wongseripipatana S, Misawa K, Kitajima M, Takayama H, Horie S. Involvement of mu-opioid receptors in antinociception and inhibition of gastrointestinal transit induced by 7-hydroxymitragynine, isolated from Thai herbal medicine Mitragyna speciosa. European journal of pharmacology. 2006; 549:63-70. [PubMed: 16978601]

Matsumoto K, Horie S, Ishikawa H, Takayama H, Aimi N, Ponglux D, Watanabe K. Antinociceptive effect of 7-hydroxymitragynine in mice: Discovery of an orally active opioid analgesic from the Thai medicinal herb Mitragyna speciosa. Life sciences. 2004; 74:2143-2155. [PubMed: 14969718]

Matsumoto K, Horie S, Takayama H, Ishikawa H, Aimi N, Ponglux D, Murayama T, Watanabe K. Antinociception, tolerance and withdrawal symptoms induced by 7-hydroxymitragynine, an alkaloid from the Thai medicinal herb Mitragyna speciosa. Life sciences. 2005; 78:2-7. [PubMed: 16169018]

Matsumoto K, Takayama H, Narita M, Nakamura A, Suzuki M, Suzuki T, Murayama T, Wongseripipatana S, Misawa K, Kitajima M, Tashima K, Horie S. MGM-9 [(E)-methyl 2-(3ethyl-7a,12a-(epoxyethanoxy)-9-fluoro-1,2,3,4,6,7,12,12b-octahydro-8-methoxy indolo[2,3a]quinolizin-2-yl)-3-methoxyacrylate], a derivative of the indole alkaloid mitragynine: a novel dual-acting mu- and kappa-opioid agonist with potent antinociceptive and weak rewarding effects in mice. Neuropharmacology. 2008; 55:154-165. [PubMed: 18550129]

Novakova L, Matysova L, Solich P. Advantages of application of UPLC in pharmaceutical analysis. Talanta. 2006; 68:908-918. [PubMed: 18970409]

Pitt JJ. Principles and applications of liquid chromatography-mass spectrometry in clinical biochemistry. The Clinical biochemist Reviews / Australian Association of Clinical Biochemists. 2009; 30:19-34. [PubMed: 19224008]

Ponglux D, Wongseripipatana S, Takayama H, Kikuchi M, Kurihara M, Kitajima M, Aimi N, Sakai S. A New Indole Alkaloid, 7 alpha-Hydroxy-7H-mitragynine, from Mitragyna speciosa in Thailand. Planta medica. 1994; 60:580-581. [PubMed: 17236085]

Takayama H, Ishikawa H, Kurihara M, Kitajima M, Aimi N, Ponglux D, Koyama F, Matsumoto K, Moriyama T, Yamamoto LT, Watanabe K, Murayama T, Horie S. Studies on the synthesis and opioid agonistic activities of mitragynine-related indole alkaloids: discovery of opioid agonists structurally different from other opioid ligands. Journal of Medicinal Chemistry. 2002; 45:19491956. [PubMed: 11960505]

Takayama H, Ishikawa H, Kurihara M, Kitajima M, Sakai S-i, Aimi N, Seki H, Yamaguchi K, Said IM, Houghton PJ. ChemInform Abstract: Structure Revision of Mitragynaline, an Indole Alkaloid in Mitragyna speciosa. ChemInform. 2001; 32:no-no.

US Food and Drug Administration. FDA Guidance for Industry: Bioanalytical Method Validation. US Department of Health and Human Services FaDA, Center for Drug Evaluation and Research; Rockville, MD: 2001.

Vuppala P, Boddu S, Furr E, McCurdy C, Avery B. Simple, Sensitive, High-Throughput Method for the Quantification of Mitragynine in Rat Plasma Using UPLC-MS and Its Application to an Intravenous Pharmacokinetic Study. Chromatographia. 2011; 74:703-710.

Watanabe K, Yano S, Horie S, Yamamoto LT. Inhibitory effect of mitragynine, an alkaloid with analgesic effect from Thai medicinal plant Mitragyna speciosa, on electrically stimulated contraction of isolated guinea-pig ileum through the opioid receptor. Life sciences. 1997; 60:933942. [PubMed: 9061050]

Watanabe, K.; Yano, S.; Horie, S.; Yamamoto, LT.; Takayama, H.; Aimi, N.; Sakai, S.; Ponglux, D.; Tongroach, P.; Shan, J.; Pang, PKT. Pharmacological properties of some structurally related indole alkaloids contained in the Asian herbal medicines, hirsutine and mitragynine, with special reference to their $\mathrm{Ca}^{2+}$ antagonistic and opioid-like effects. In: Watanabe, H.; Shibuya, T.; 
Farnsworth, NR., editors. Pharmacological Research on Traditional Herbal Medicines. Tokyo: Harwood Academic Press; 1999. p. 163-177. 
(a)

(b)<smiles>CCC1CN2CCc3c([nH]c4cccc(OC)c34)[C@H]2C[C@H]1/C(=C\OC)C(=O)OC</smiles>

(c)
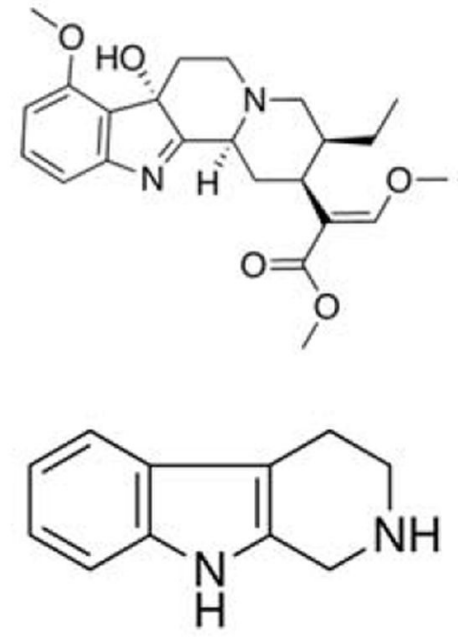

Figure 1.

Chemical structures of mitragynine (a), 7-hydroxymitragynine (b) and tryptoline (c). 

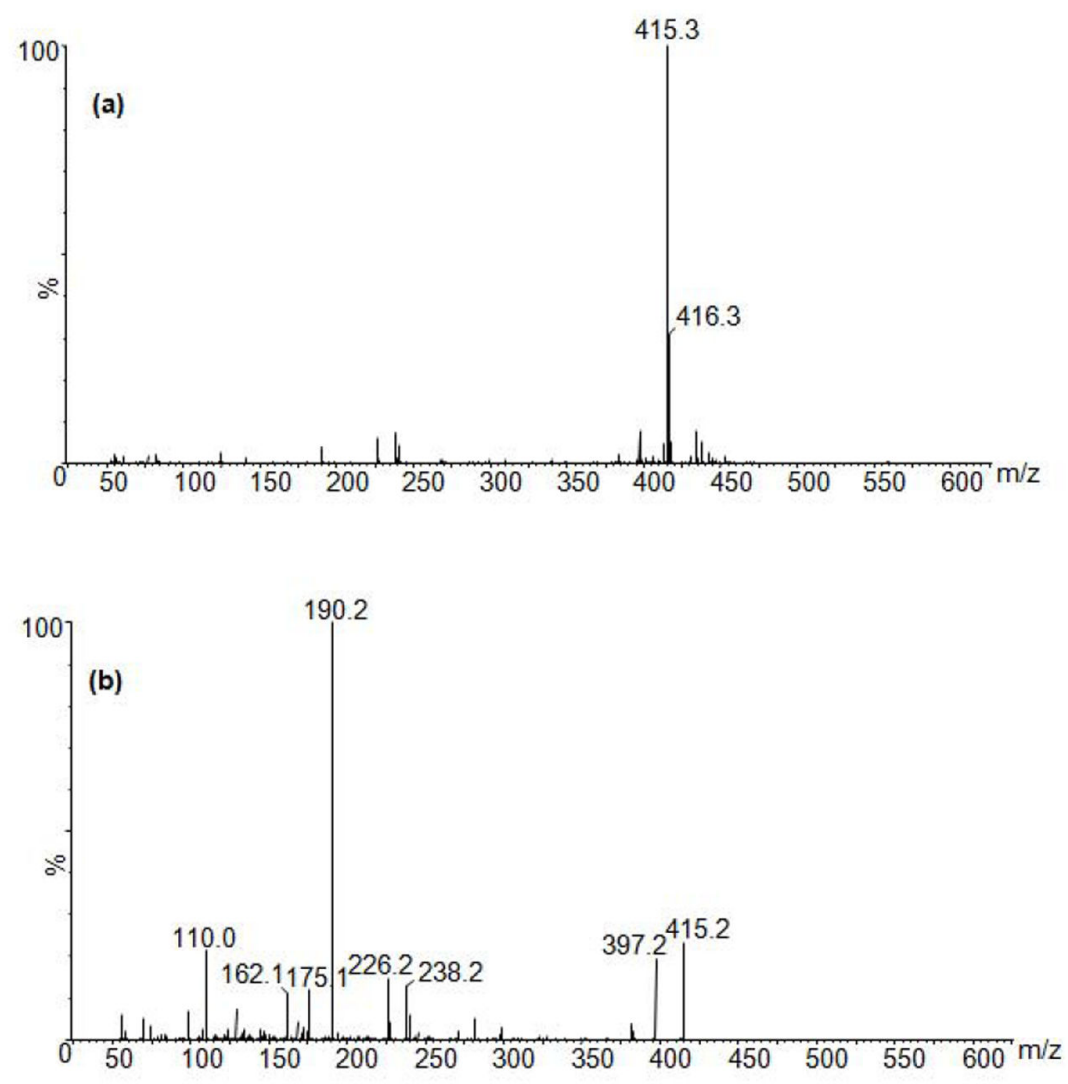

Figure 2.

Protonated molecular ion spectra (a) and product ion spectra (b) of 7-hydroxymitragynine. 

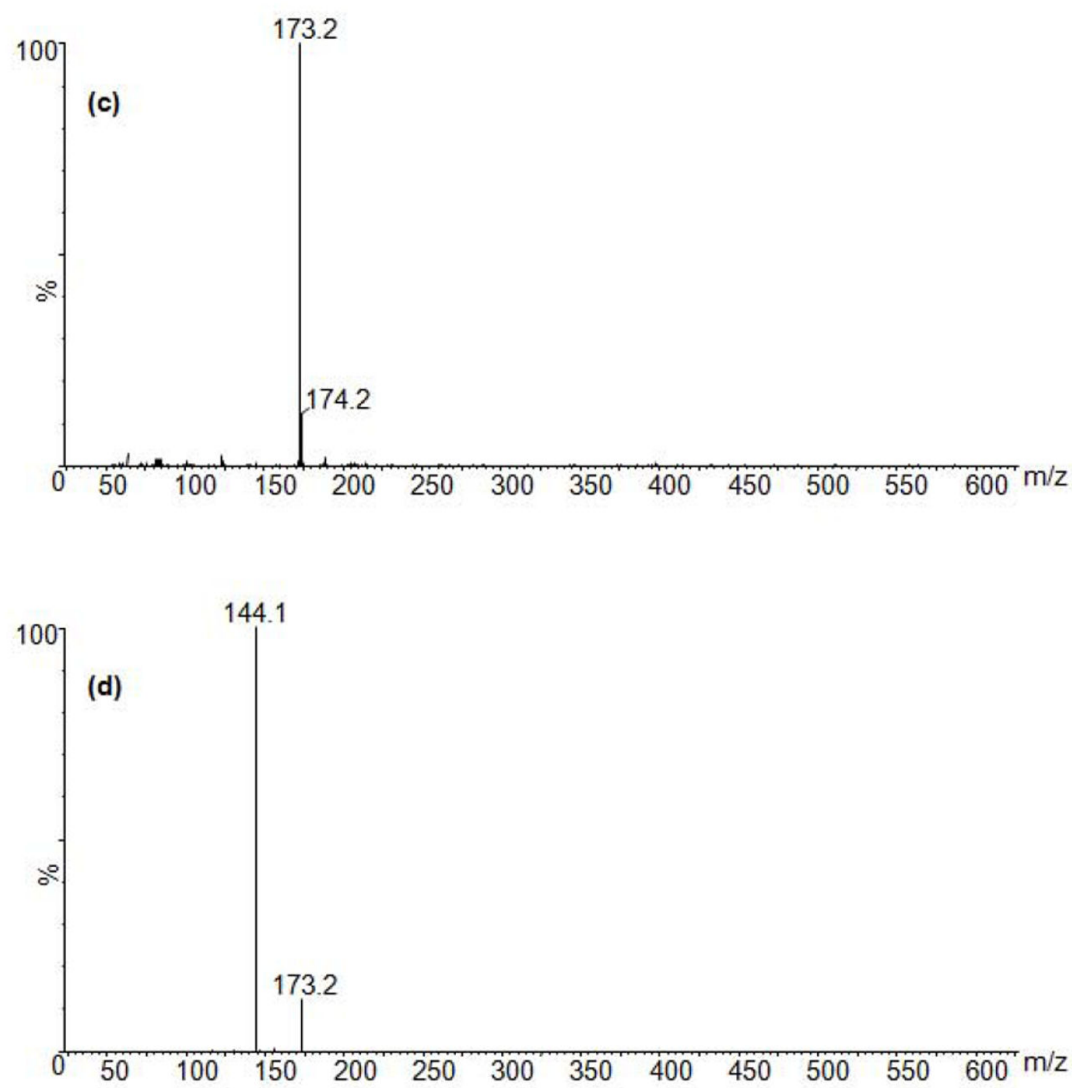

Figure 3.

Protonated molecular ion spectra (a) and product ion spectra (b) of tryptoline. 

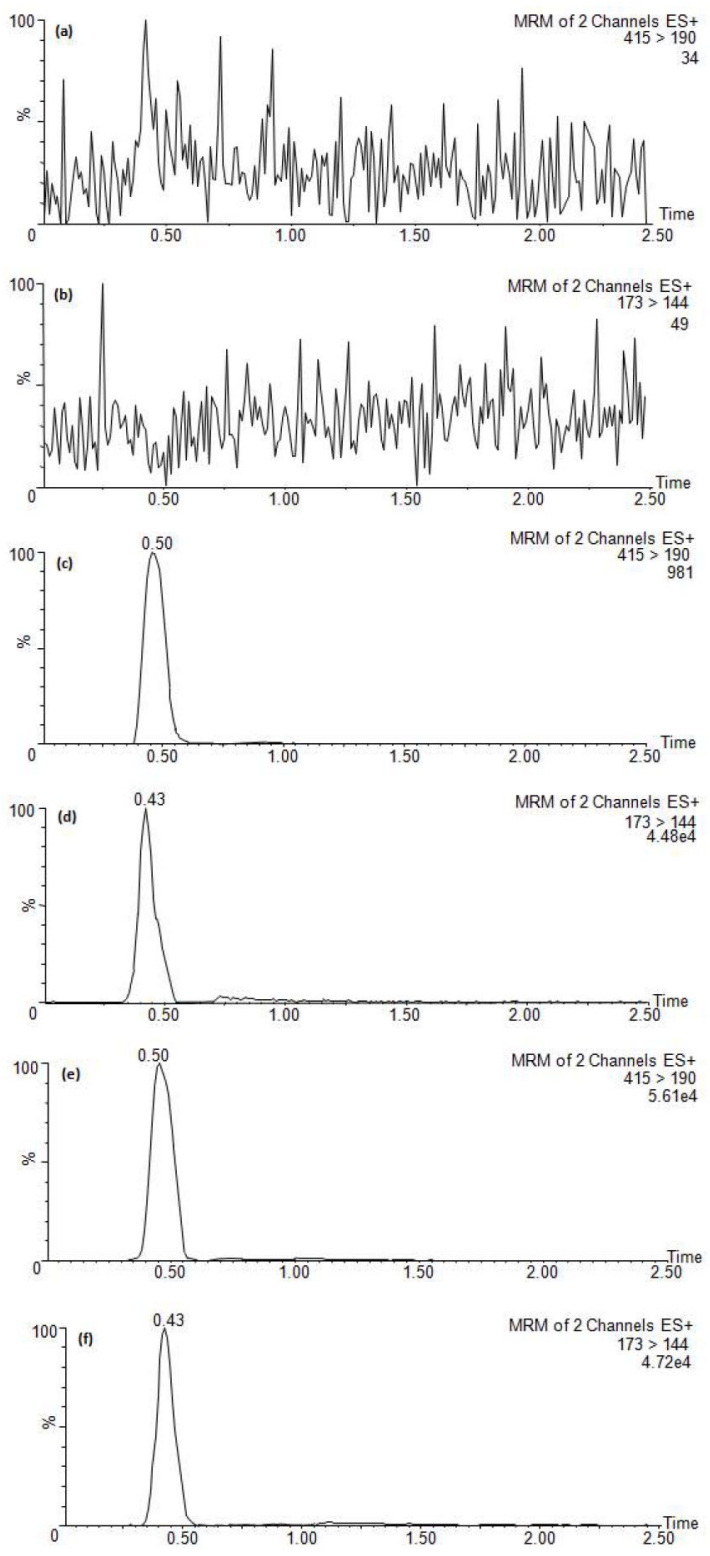

Figure 4.

(a) Chromatogram for blank plasma at $\mathrm{m} / \mathrm{z} 415 \rightarrow 190$ (for 7-hydroxymitragynine); (b) chromatogram for blank plasma at $\mathrm{m} / \mathrm{z} 173 \rightarrow 144$ (for internal standard); (c) chromatogram for blank plasma spiked with $10 \mathrm{ng} / \mathrm{mL}$ 7-hydroxymitragynine; (d) chromatogram for blank plasma spiked with $100 \mathrm{ng} / \mathrm{mL}$ IS; and (e, f) chromatogram for plasma sample 10min after intravenous administration spiked with IS. 


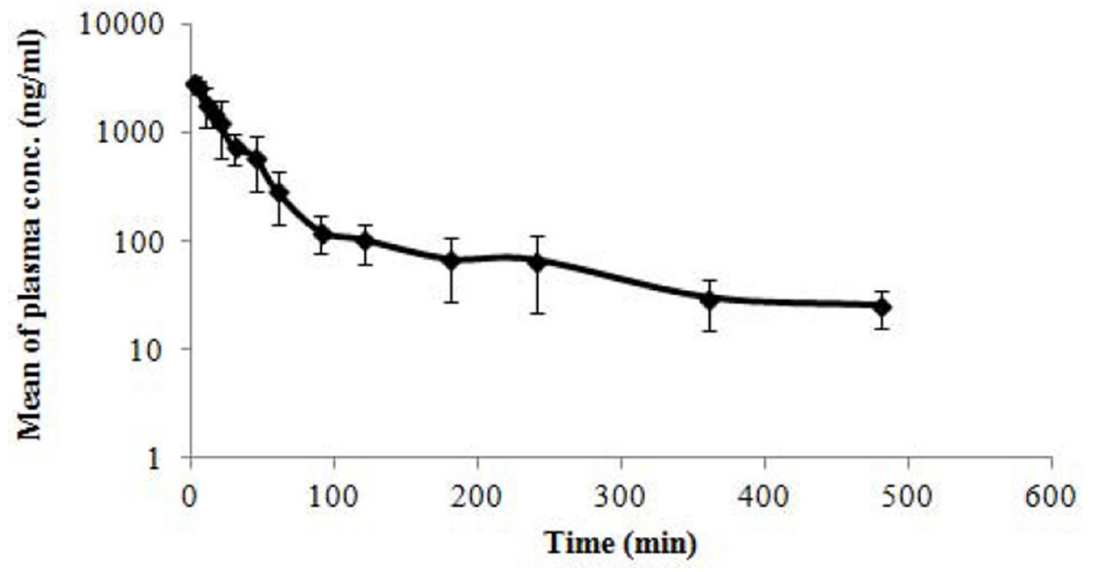

Figure 5.

Mean concentration of 7-hydroxymitragynine in plasma after i.v. administration vs time profile $(n=6)$. 


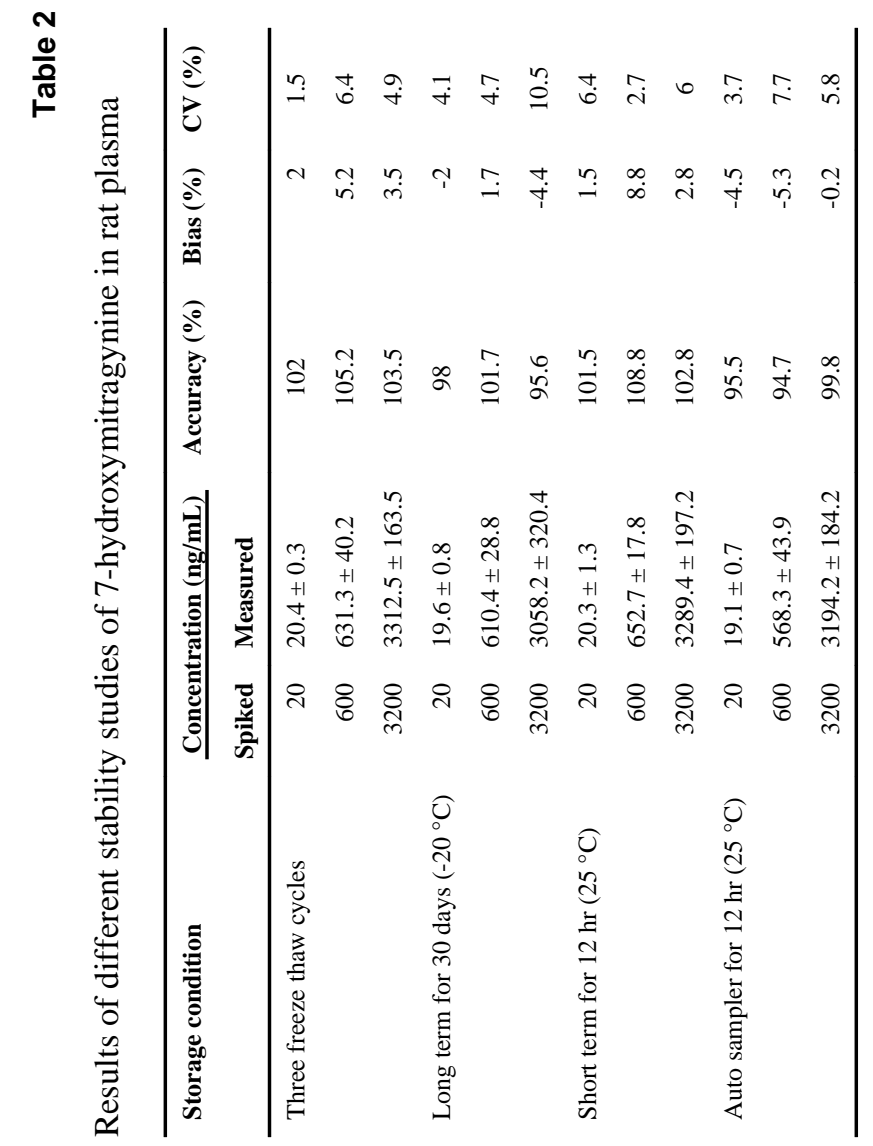


Table 3

Pharmacokinetic parameters after intravenous administration of $4 \mathrm{mg} / \mathrm{kg}$ 7-OHMG to male Sprague Dawley rats $(n=6)$

\begin{tabular}{|ll|}
\hline Parameter & Mean \pm SD \\
\hline $\mathrm{T}_{1 / 2}(\mathrm{~min})$ & $22.9 \pm 3.6$ \\
$\mathrm{C}_{\max }(\mu \mathrm{g} / \mathrm{mL})$ & $3.0 \pm 0.3$ \\
$\mathrm{AUC}_{0 \rightarrow \infty}(\mu \mathrm{g} \mathrm{min} / \mathrm{mL})$ & $98.3 \pm 32.1$ \\
$\mathrm{~V}_{\mathrm{d}}(\mathrm{mL} / \mathrm{kg})$ & $1595.8 \pm 586.3$ \\
$\mathrm{CL}(\mathrm{mL} / \mathrm{min} / \mathrm{kg})$ & $44.2 \pm 14.8$ \\
$\mathrm{MRT}(\mathrm{min})$ & $70.9 \pm 10.0$ \\
\hline
\end{tabular}

